\title{
HST/STIS observations of sdBV stars: testing diffusion and pulsation theory
}

\author{
S.J. O'Toole ${ }^{1}$, U. Heber ${ }^{1}$, P. Chayer ${ }^{2}$, G. Fontaine ${ }^{3}$, D. O'Donoghue ${ }^{4}$, \\ S. Charpinet ${ }^{5}$ \\ ${ }^{1}$ Dr Remeis-Sternwarte, Astronomisches Institut der Universität \\ Erlangen-Nürnberg, Sternwartstr. 7, Bamberg D-96049, Germany \\ ${ }^{2}$ Department of Physics and Astronomy, Johns Hopkins University, \\ 3400 N. Charles St., Baltimore MD 21218, USA \\ ${ }^{3}$ Département de Physique, Université de Montréal, CP 6128, Station \\ Centreville, Montréal, QCH3C-3J7, Canada \\ ${ }^{4}$ South African Astronomical Observatory, PO Box 9, Observatory \\ 7935, South Africa \\ ${ }^{5}$ Laboratoire d'Astrophysique, Observatoire Midi Pyrénées, 14 avenue \\ Edouard Belin, 31400 Toulouse, France
}

\begin{abstract}
We present the initial results of an abundance analysis of echelle UV spectra of 5 hot subdwarf B ( $\mathrm{sdB}$ ) stars, which form part of a study to test pulsation and diffusion calculations. SdB stars have been identified as core helium-burning objects on the extreme Horizontal Branch. Around $5 \%$ of sdB stars show short-period acoustic mode oscillations, which models predict are due to an opacity bump caused by the ionisation of iron group elements. The necessary metal abundance has to be maintained by diffusive equilibrium between gravitational settling and radiative levitation. However, analyses of high resolution optical spectra has revealed that we cannot discriminate between pulsating and nonpulsating sdB stars on the basis of the surface iron abundance. Our initial analysis of HST/STIS observations of 3 pulsators and 2 non-pulsators in the near and far UV suggests this may also be the case for other iron group elements, although further work will be needed to verify this.
\end{abstract}

\section{Why do some sdB stars pulsate while others do not?}

Pulsations in subdwarf B (sdB) stars provide an opportunity to use the tools of asteroseismology to probe their interiors. Modelling suggests that the sdB pulsations are driven by an opacity bump due to iron and other metallic species (see Charpinet et al. 2001 for a review). The models require a sufficient abundance of $\mathrm{Fe}$ at an appropriate level in the envelope, and an abundance which can be maintained by diffusive equilibrium between gravitational settling and radiative levitation. While they give rise to pulsations in hot sdB stars, the models do not yet explain why, when given two spectroscopically similar stars, one will pulsate and the other will not. This overlap in the $\log g-T_{\text {eff }}$ plane was demonstrated by Koen et al. (1999). Fontaine \& Chayer (1997) suggest different mass-loss 

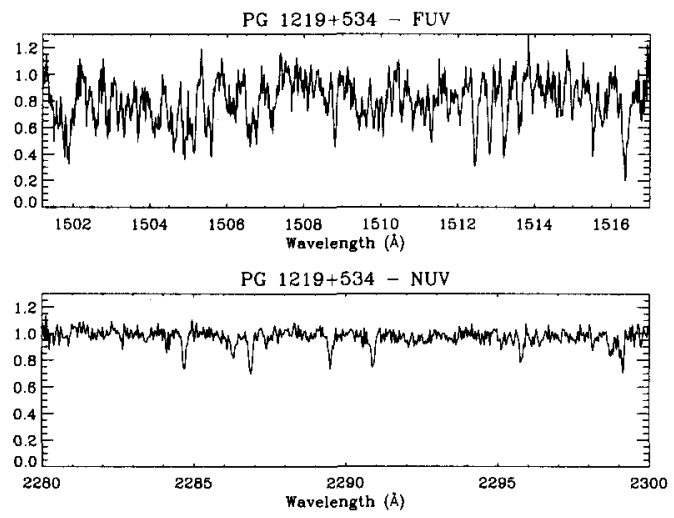

Figure 1. A comparison of the FUV (top) and NUV (bottom) spectra for PG $1219+534$. Note that the FUV spectrum is covered by a forest of lines, whereas for the NUV the continuum can be traced well. Therefore abundance analysis is much more difficult for the FUV than for the NUV spectra.

rates as one possible explanation, whereby a star with a higher mass-loss rate may have a depleted Fe abundance, and therefore not show pulsations.

Using HST/STIS UV spectra, our goal is to measure the metal abundances of 3 pulsating sdB stars and 2 non-pulsating sdB stars, and to determine whether the non-pulsators' abundances are significantly different from those of the pulsators. With these observations we can derive abundances for elements not observable from the ground, e.g. the iron group. If there are no significant differences between the abundances patterns, then this may point to the discriminating factor being stellar winds as suggested by Fontaine \& Chayer. The abundances resulting from this study will also provide an important test of the predictions of diffusion theory.

\section{Observations with HST}

Observations were made using the Space Telescope Imaging Spectrograph (STIS) onboard HST. We used medium resolution gratings in echelle mode in both the far UV (grating E140M) and the near UV (grating E230M). The FUV spectrum covers the wavelength range $1149-1730 \AA$, while the NUV covers the range $1635-2365 \AA$. Three pulsators were observed, PG 1219+534, Feige 48, and PG $1605+072$, the latter showing rapid rotation with $v \sin i=39 \mathrm{~km} \mathrm{~s}^{-1}$ (Heber et al. 1999). The two non-pulsators, Ton S-227 and Feige 66, are very similar spectroscopically to the pulsator PG $1219+534$ - Ton S-227 is almost its spectroscopic twin. 

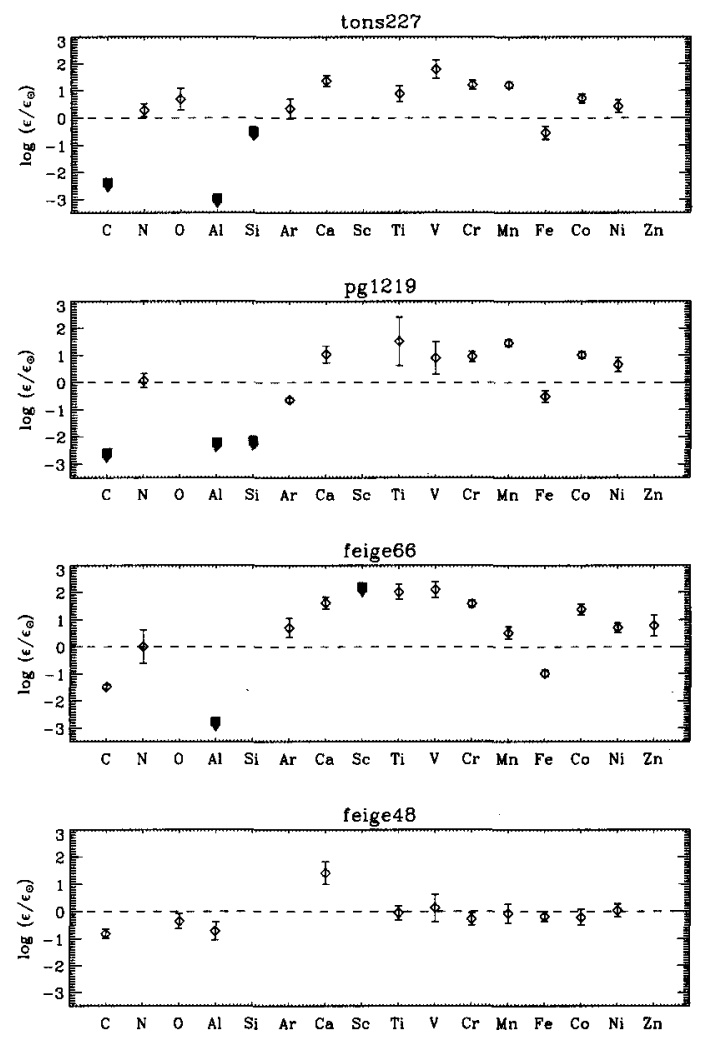

Figure 2. Abundances for four sdB stars derived from near UV HST/STIS spectra. The high $\log g$ stars (top three panels) all show enriched iron group abundances, while Feige 48 (bottom panel) shows only solar iron group abundances. Note that PG $1219+534$ (a pulsator) and Ton S-227 (a non-pulsator) have almost identical abundances. The filled symbols represent upper limits.

\section{Preliminary results}

In order to overcome the substantial line blending in the spectra, we used a solar metallicity model and adjusted the abundances of each element until $\chi^{2}$ was minimised. This was done using Michael Lemke's version of the LINFOR program (originally developed by Holweger, Steffen, and Steenbock at Kiel University). Oscillator strengths were taken from the Kurucz line list, as were damping constants for all metal lines. For all of the results presented here, the microturbulent velocity was set to $0 \mathrm{~km} \mathrm{~s}^{-1}$. The current study is limited to the near UV spectra, since for these spectra it was easier to determine a continuum level (see Fig. 1). Abundances will be derived from the far UV spectra and for PG $1605+072$ (whose spectra are complicated by rotation) in the near future. The results for the four studied stars are shown in Fig. 2. Note that the errors may be underestimated because of difficulties determining the continuum level. 
From our preliminary analysis, we find no significant differences between abundances of pulsating and non-pulsating sdB stars. In particular the abundances of PG $1219+534$ and Ton S-227 are remarkably similar. There is a noticeable difference between the abundance pattern of Feige 48 and the other 3 stars. The hotter, higher surface gravity stars all show super-solar iron group abundances, while for Feige 48 these elements have solar abundances. The element that stand out is calcium, which is super-solar in all 4 stars. These effects appear to be due to "selective diffusion", i.e. the interplay between radiative levitation and gravitational settling. It is yet to be seen whether diffusion models can explain these effects.

\section{Conclusions and the future}

As a consistency check, we have compared our results with previous analyses of these stars. For Feige 48 and PG 1219+534, Heber et al. (2000) used highresolution spectra to measure abundances (or set upper limits) for six of the species detectable in our spectra. A slightly lower Fe abundance is the only noticeable difference between our results and that of Heber et al. All other elements are in reasonable agreement. This lower Fe abundance is also seen in FUSE spectra (Chayer et al. 2003). It is difficult to compare our results for Feige 66 with those of Baschek et al. (1982) because of the relative inaccuracy of the atomic data available at that time. However, we can say that, apart from calcium, the agreement appears to be reasonably good. Overall we believe that our analysis is consistent with previous analyses.

All of the high $\log g$ stars have super-solar iron group element abundances, and in particular PG $1219+534$ and Ton S-227 appear to be very similar, while Feige 48 , with a substantially lower $\log g$, has approximately solar abundances. Do these results present a problem for the Charpinet et al. Fe driving model? At this stage we do not want to draw any premature conclusions: more abundance analyses of pulsating and non-pulsating sdB stars are needed to see any heavy element abundance patterns (e.g., Chayer et al., in preparation). High resolution optical and FUV observations or more sdB stars should clarify the driving mechanism.

\section{References}

Baschek, B., Hoflich, P., Scholz, M. 1982, A\&A112, 76

Charpinet, S., Fontaine, G., Brassard, P. 2001, PASP113, 775

Chayer, P., et al. 2003, in Extreme Horizontal Branch Stars and related objects, to be published by Kluwer

Fontaine, G., Chayer, P. 1997, in 3rd Conference on Faint blue stars, Schenectady, L. Davis press, p. 169

Heber, U., Reid, I.N., Werner, K. 2000, A\&A363, 198

Koen, C., O'Donoghue, D., Kilkenny, D., Stobie, R.S., Saffer, R.A., 1999, MNRAS306, 213 